\title{
Effect of ramelteon coadministered with antidepressant in patients with insomnia and major depressive disorder: an exploratory study
}

\author{
Naohisa Uchimura ${ }^{1} \cdot$ Keisuke Nakatome ${ }^{2} \cdot$ Kouji Miyata $^{2} \cdot$ Makoto Uchiyama $^{3}$
}

Received: 6 March 2019 / Accepted: 4 July 2019 / Published online: 13 July 2019

(c) The Author(s) 2019

\begin{abstract}
The purpose is to evaluate the effect and safety of ramelteon $8 \mathrm{mg} /$ day for 8 weeks in the treatment of insomnia in patients with concurrent depression in an exploratory manner. This phase 4, open-label, exploratory study included outpatients aged 20 to $<65$ years with sleep-onset insomnia and major depressive disorder taking stable antidepressant medication. Following a 1-week run-in, 26 eligible patients received ramelteon $8 \mathrm{mg} /$ day plus their usual antidepressants for 8 weeks. Outcomes included sleep parameters measured by actigraphy and sleep diary, 3-Dimensional Sleep Scale (3DSS), 17-item Hamilton Rating Scale for Depression (HAM-D17), Patient Global Impression (PGI), adverse events, and body weight. Actigraphy- and diary-measured sleep latency improved at the end of ramelteon treatment (mean decrease -6.8 and -11.5 min, respectively), but neither change reached statistical significance in this exploratory study. Other subjective measures indicated improved sleep, including diary-measured total sleep time (mean change $+41.2 \mathrm{~min} ; p=.0220$ ) and number of nocturnal awakenings $(-.4 ; p=.0420)$, and 3DSS total scores for sleep quality and quantity $(p<.01)$. Most patients $(88.5 \%)$ reported improvement in PGI. HAM-D17 total scores improved at end of treatment (mean change $-4.0 ; p<.0001$ ). One patient discontinued ramelteon due to moderate somnolence. Ramelteon coadministered with antidepressants was well tolerated. Results from this exploratory study suggest that ramelteon may be effective and well tolerated in the treatment of sleep-onset insomnia in patients with concurrent depression.
\end{abstract}

Keywords Actigraphy $\cdot$ Antidepressants $\cdot$ Depressive disorder $\cdot$ major $\cdot$ Insomnia $\cdot$ Ramelteon

\section{Introduction}

Insomnia is a common medical condition in Japan, with clinical and social consequences [1]. The prevalence of depression in Japan is rising, partly in association with increasing workplace demands, and poses a social and economic burden [2-4]. Insomnia and depression often coexist [5], and treatment of insomnia symptoms has been reported to improve the response to antidepressants [6]. Insomnia is more prevalent with increasing age and lifestyle factors such

Keisuke Nakatome

keisuke.nakatome@takeda.com

1 Department of Neuropsychiatry, Kurume University School of Medicine, Kurume, Japan

2 Japan Medical Office, Takeda Pharmaceutical Company Limited, 1-1 Nihonbashi-Honcho 2-chome, Chuo-ku, Tokyo 103-8668, Japan

3 Department of Psychiatry, Nihon University School of Medicine, Tokyo, Japan as obesity, but is also associated with an altered circadian rhythm due to an irregular sleep schedule, and anxiety about sleep expectations. Depression, which is typically associated with anxiety, is now known to also be exacerbated by an irregular sleep schedule. Thus, insomnia and depression have some common risk factors, which may explain the frequent comorbidity of these conditions [7, 8].

The benzodiazepine and nonbenzodiazepine hypnotics, which act as gamma-aminobutyric acid (GABA)A receptor agonists, have long been the treatments of choice for insomnia $[9,10]$. However, both classes exert sedative and anxiolytic effects, and are associated with next-day sedation, cognitive deficits such as anterograde amnesia, rebound withdrawal symptoms of anxiety and insomnia, and risk of dependence $[10,11]$. Ramelteon is a novel melatonin receptor agonist with high selectivity for the melatonin MT1/ MT2 receptors [12]. Preclinical and clinical studies have shown that ramelteon is associated with sleep promotion and can accelerate circadian rhythm re-entrainment, leading to the improvement of both transient and chronic insomnia 
Fig. 1 Study design. $W k$ week

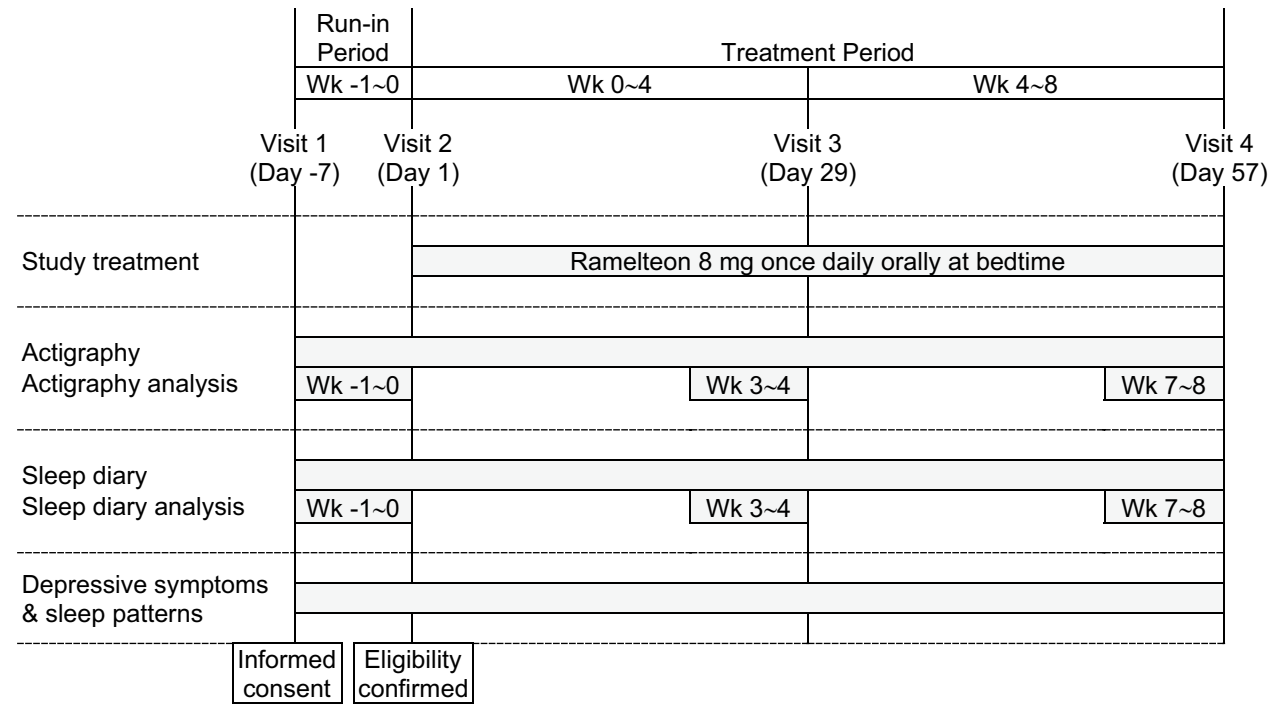

without sedative or anxiolytic effects [12-17]. Ramelteon is approved in Japan and used for the treatment of insomnia characterized by difficulty falling asleep [18]. Ramelteon's lack of sedative and anxiolytic properties may be clinically important, particularly in the treatment of patients with insomnia and concurrent depression.

There are limited reports on the efficacy and safety of ramelteon in patients with insomnia and depression or other psychiatric diseases [19, 20], and none of the reported studies used objective measures. The present study is thus designed to evaluate the efficacy of ramelteon in the treatment of insomnia in patients with concurrent depression, using both objective and subjective measurements in an exploratory manner.

\section{Materials and methods}

\section{Study design}

This was a phase 4, multicenter, single-arm, open-label, exploratory study to measure the effect of ramelteon using actigraphy and a sleep diary in patients with insomnia and major depressive disorder. The study included a 1-week runin period when patients continued their usual antidepressant medication and were instructed to keep a daily sleep diary and continuously wear an actigraph device. Patients who were eligible at the end of the run-in period then entered the 8 -week treatment period and received $8 \mathrm{mg}$ ramelteon once daily in addition to their usual antidepressant medication, and continued to keep a sleep diary and wear an actigraph. The use of fluvoxamine and insomnia medications other than ramelteon was prohibited during the study. Patient visits occurred at the start of the run-in period, at the start of the treatment period (baseline), and at weeks 4 and 8 of the treatment period (Fig. 1).

The study was conducted at 7 medical institutions throughout Japan between May 2017 and January 2018. The study was approved by the ethics committees of each study site and conducted in compliance with Japanese Ethical Guidelines for Medical and Health Research Involving Human Subjects, the International Conference on Harmonisation guidelines defining Good Clinical Practice, and the Declaration of Helsinki. All patients provided written informed consent. The study was sponsored by Takeda Pharmaceutical Company Limited, and is registered at clinicaltrials.gov (NCT02669082) and the Japan Pharmaceutical Information Center (JapicCTI-163143).

\section{Study population}

Eligible patients were male and female outpatients aged 20 to $<65$ years, with sleep-onset insomnia on at least 3 days per week for at least 4 weeks before giving informed consent, with a routine sleep schedule defined as bedtime between 21:00 and 01:00 on at least 4 days per week. Eligible patients also had depression, diagnosed according to Diagnostic and Statistical Manual of Mental Disorders Fifth Edition (DSM-5), and were on stable antidepressant medication, with no change of antidepressant medication or dosage for at least 4 weeks before the run-in period.

At the start of the run-in period and the start of the treatment period, patients were to have a total score of $\leq 16$ on the 17-item Hamilton Rating Scale for Depression (HAMD17), indicating mild depression, as well as either an HAMD17 "Insomnia Early" score of 2, or an HAM-D17 score of 1 for "Insomnia Early" and subtotal score of $\geq 3$ for "Insomnia Middle" and "Insomnia Late". During the run-in period, patients were to have sleep latency of $\geq 30 \mathrm{~min}$ and total 
nocturnal sleep time of $\leq 6.5 \mathrm{~h}$ on $\geq 3$ days, measured by actigraphy.

Patients were ineligible to enter the study if they had psychiatric or neurological diseases other than depression that could affect sleep status; were shift workers or night workers; took oral ramelteon within 4 weeks before informed consent; or took any oral insomnia medications within 2 weeks before the treatment period. Patients were also ineligible if they had an HAM-D17 "Suicide" score of $\geq 1$ at the start of the run-in period or treatment period, or had made any suicide attempts within 24 weeks prior to or during the run-in period. Patients were excluded if they had severe liver disorder, or were pregnant, nursing, or planning to become pregnant or donate eggs up to 4 weeks after the end of the study.

\section{Treatment protocol}

Patients who were eligible at the end of the run-in period received ramelteon (Rozerem ${ }^{\circledR}$; Takeda Pharmaceutical Company Limited), a novel melatonin receptor agonist approved for the treatment of insomnia characterized by difficulty with sleep onset. Patients were to take one $8 \mathrm{mg}$ ramelteon tablet once daily at bedtime for 8 weeks.

Patients were also to continue their usual oral antidepressant medication without any change to the dosage regimen during the study. The addition of antidepressants was not allowed from 4 weeks before the run-in period.

Insomnia medications other than ramelteon were not to be used and non-pharmacological insomnia treatments were not to be added or changed during the study.

\section{Outcome measures}

Patient rest and physical activity were measured objectively with an FS-750 actigraph (Acos Company Limited, Nagano, Japan) worn throughout the study. The device was worn at the waist, as it was less affected by movement artifacts than actigraphs worn at the wrist [21]. Actigraphy data analysis software (SleepSign Act; Kissei Comtec Company Limited, Nagano, Japan) was used to calculate sleep latency, total sleep time (TST; total time in bed minus sleep latency, wake after sleep onset [WASO], and time from waking to leaving the bed), WASO, the number of nocturnal awakenings, sleep efficiency ([TST/total time in bed] $\times 100$ ), and daytime activity level (step counts). For each of these parameters, the validity of the measured data was checked according to pre-specified procedures. Sleep parameters were calculated for baseline, week 4 , and week 8 using the mean value from the previous 7 days.

Sleep parameters were assessed subjectively using a sleep diary in which patients recorded sleep latency, bedtime hour, awakening hour, and number of nocturnal awakenings throughout the study. The TST was calculated as total time in bed (awaking hour minus bedtime hour) minus sleep latency; thus, TST based on the sleep diary included time awake during the night and, therefore, differed from TST using actigraphy, which excluded WASO and time from waking to leaving the bed.

The 3-Dimensional Sleep Scale (3DSS), a patient-administered questionnaire developed in Japan, was used to assess sleep dimensions and phases [22]. The 3DSS consists of 15 questions that assess 3 sleep dimensions: sleep phase (i.e., regularity), sleep quality, and sleep quantity. Each question is answered using a four-point scale ( $0-3)$, providing a total score ranging from 0 to 15 for each dimension, with higher scores indicating better sleep. Additional outcomes included the HAM-D17 questionnaire, used to rate the severity of depression based on an interview with the patient [23], and the Patient Global Impression (PGI) scale, completed by the patient to rate improvement or worsening of their condition. The 3DSS and HAM-D17 questionnaires were completed at baseline, and weeks 4 and 8 of the treatment period, and the PGI was completed at the end of treatment. Safety assessments included adverse events and body weight.

\section{Statistical analysis}

All patients who received at least one dose of ramelteon were included in the full analysis set, which was used for the analysis of efficacy and safety data. The target sample size of 30 patients was based on feasibility. No formal power calculation was performed, because no hypothesis was being tested.

Data were summarized using descriptive statistics. The primary endpoint was change in actigraphy-measured sleep latency from baseline (defined as the start of the treatment period) to end of treatment, using the mean value from the previous 7 days. Observed and percentage changes from baseline were analyzed using the 1-sample $t$ test. Secondary outcomes measured by actigraphy or sleep diary were analyzed in the same way as the primary endpoint. Correlation between actigraphy-measured and diary-measured values was calculated using the Spearman correlation coefficient for sleep latency and TST at end of treatment. Subgroup analyses for the primary endpoint were performed for the previous use of hypnotics (benzodiazepine, nonbenzodiazepine, other hypnotic, or none), concomitant use of benzodiazepine anxiolytics (yes, no), concomitant use of selective serotonin reuptake inhibitors (SSRIs) or serotonin and norepinephrine reuptake inhibitors (SNRIs; yes, no), duration of insomnia ( $<3$ and $\geq 3$ years), duration of depression ( $<3$ and $\geq 3$ years), and 3DSS sleep phase total score at baseline $(<9$ and $\geq 9)$. Observed and percentage changes from baseline to end of treatment were also summarized for HAM-D17 (total, sleep-related, and non-sleep-related 
Table 1 Baseline characteristics

\begin{tabular}{ll}
\hline Variable & $\begin{array}{l}\text { Ramelteon } 8 \mathrm{mg} \\
(N=26)\end{array}$ \\
\hline Age, years & $36.9 \pm 8.04$ \\
Male, $n(\%)$ & $19(73.1)$ \\
Duration of insomnia, years & $4.6 \pm 5.35$ \\
Duration of depression, years & $3.6 \pm 4.58$ \\
Previous use of hypnotics, $n(\%)$ & $18(69.2)$ \\
Benzodiazepine & $6(23.1)$ \\
Nonbenzodiazepine & $6(23.1)$ \\
Other hypnotic & $8(30.8)$ \\
Concomitant antidepressants, $n(\%)$ & $26(100)$ \\
Tricyclic antidepressant & $4(15.4)$ \\
SSRI & $11(42.3)$ \\
SNRI & $10(38.5)$ \\
Noradrenergic and specific serotonergic antide- & $3(11.5)$ \\
pressant & \\
Other antidepressant & $4(15.4)$ \\
Atypical antipsychotic & $4(15.4)$ \\
Benzodiazepine anxiolytic & $11(42.3)$ \\
Sleep latency, minutes & \\
Actigraphy & $44.8 \pm 20.29$ \\
Sleep diary & $51.2 \pm 51.96$ \\
Total sleep time, minutes ${ }^{\mathrm{a}}$ & \\
Actigraphy & $314.9 \pm 74.29$ \\
Sleep diary & $408.0 \pm 72.15$ \\
HAM-D17 total score & $9.2 \pm 2.12$ \\
\hline & \\
\hline
\end{tabular}

Variables are presented as $n(\%)$ or mean \pm standard deviation

HAM-D17 17-Item Hamilton Rating Scale for Depression, SNRI serotonin and norepinephrine reuptake inhibitor, SSRI selective serotonin reuptake inhibitor

${ }^{a}$ Actigraphy-measured total sleep time was calculated as total time in bed minus sleep latency, wake after sleep onset, and time from waking to leaving the bed. Sleep diary-measured total sleep time was calculated as total time in bed (awaking hour minus bedtime hour) minus sleep latency

scores) and 3DSS scores, and analyzed using the 1-sample $t$ test. Missing data were not imputed or replaced.

\section{Results}

\section{Demographic and baseline clinical characteristics}

Of 36 patients who were screened in the run-in period, 26 patients entered the treatment period. Of these, 25 patients completed the study and 1 patient discontinued ramelteon due to an adverse event. All 26 patients were included in the full analysis set.

Most patients $(73.1 \%)$ were male, the mean age was 36.9 years, and the mean duration of insomnia and depression was 4.6 and 3.6 years, respectively (Table 1). At baseline, mean sleep latency was 44.8 min measured by actigraphy and 51.2 min by sleep diary. Mean HAM-D17 total score was 9.2 and $69.2 \%$ of patients had previously used hypnotic medication. All patients took at least one concomitant antidepressant during the study; the most commonly used were SSRIs (42.3\%), benzodiazepine anxiolytics (42.3\%), and SNRIs (38.5\%).

\section{Sleep parameters}

Improvements in sleep latency at end of treatment were measured by both actigraphy (mean $\pm \mathrm{SD}$ decrease $-6.8 \pm 32.50 \mathrm{~min} ; p=.2955$ ) and sleep diary $(-11.5 \pm 38.09 \mathrm{~min} ; p=.1358)$, but the decreases did not reach statistical significance (Table 2). Improvements in TST at end of treatment were measured by both actigraphy (mean \pm SD increase $24.2 \pm 87.47 \mathrm{~min} ; p=.1706$ ) and sleep diary $(41.2 \pm 85.97 \mathrm{~min} ; p=.0220)$, with the improvement in diary-measured TST reaching statistical significance. Improvements were evident by week 4 with both methods for sleep latency (mean \pm SD decrease $-7.2 \pm 31.73$ min by actigraphy $[p=.2561]$ and $-12.7 \pm 37.11 \mathrm{~min}$ by sleep diary $[p=.0934]$ ) and TST (mean \pm SD increase $32.5 \pm 77.41 \mathrm{~min}$ by actigraphy $[p=.0422]$ and $32.2 \pm 94.18 \mathrm{~min}$ by sleep diary $[p=.0933])$, with the change in actigraphy-measured TST at week 4 reaching statistical significance. Most patients (65.4\%-69.2\%) had improvements in sleep latency or TST of $\geq 1 \mathrm{~min}$ at the end of treatment, whether measured by actigraphy or sleep diary (Table 2).

There was a positive correlation between the actigraphy and sleep diary methods for the measurement of sleep latency (Spearman $r=.5319[p=.0051])$ and TST $(r=.3600$ $[p=.0708])$. However, the mean $( \pm \mathrm{SD})$ number of nocturnal awakenings was consistently higher when measured by actigraphy $(6.1 \pm 2.62$ at baseline and $6.6 \pm 2.63$ at end of treatment $)$ than by sleep diary $(1.9 \pm 1.08$ and $1.4 \pm 1.03$, respectively), and showed a mean $( \pm S D)$ increase from baseline to end of treatment by actigraphy $(.5 \pm 1.34$; $p=.0534)$ compared with a decrease $(-.4 \pm 1.05 ; p=.0420)$ by sleep diary.

Sleep efficiency and WASO were measured only by actigraphy. Sleep efficiency improved at the end of treatment (mean \pm SD increase $1.83 \pm 11.20 \%$ ), whereas WASO increased $(10.1 \pm 38.74 \mathrm{~min})$, but neither change was statistically significant. Daytime activity level, based on actigraphy-measured step count, showed a numerical increase at the end of treatment (mean \pm SD increase 111.1 \pm 2418.19 steps) that was not statistically significant.

\section{Sleep latency subgroup analysis}

Subgroup analyses were performed for sleep latency measured by actigraphy (Fig. 2). Improvement in 
Table 2 Change in sleep latency and total sleep time

\begin{tabular}{lccccc}
\hline Endpoint & \multicolumn{2}{l}{ Ramelteon $8 \mathrm{mg}(N=26)$} & & \\
\cline { 2 - 6 } & Baseline & End of treatment & Change from baseline & $p$ value $^{\mathrm{a}}$ & $\begin{array}{l}\text { Patients with } \\
\text { improvement } n \\
(\%)^{\mathrm{b}}\end{array}$ \\
\hline Actigraphy-measured parameters, minutes & & & \\
Sleep latency & $44.8 \pm 20.29$ & $38.0 \pm 37.03$ & $-6.8 \pm 32.50$ & .2955 & $18(69.2)$ \\
Total sleep time & $314.9 \pm 74.29$ & $339.1 \pm 84.62$ & $24.2 \pm 87.47$ & .1706 & $17(65.4)$ \\
Diary-measured parameters, minutes & & & & \\
Sleep latency & $51.2 \pm 51.96$ & $39.7 \pm 37.18$ & $-11.5 \pm 38.09$ & .1358 & $18(69.2)$ \\
Total sleep time $^{\mathrm{c}}$ & $408.0 \pm 72.15$ & $449.2 \pm 83.98$ & $41.2 \pm 85.97$ & .0220 & $18(69.2)$ \\
\hline
\end{tabular}

Variables are presented as mean \pm standard deviation or $n(\%)$

${ }^{a}$ One-sample $t$ test for change from baseline to end of treatment

${ }^{\mathrm{b}}$ Improvement in sleep latency was defined as a decrease of $\geq 1 \mathrm{~min}$ from baseline to end of treatment; improvement in total sleep time was defined as an increase of $\geq 1 \mathrm{~min}$ from baseline to end of treatment

${ }^{\mathrm{c}}$ Actigraphy-measured total sleep time was calculated as total time in bed minus sleep latency, wake after sleep onset, and time from waking to leaving the bed. Sleep diary-measured total sleep time was calculated as total time in bed (awaking hour minus bedtime hour) minus sleep latency

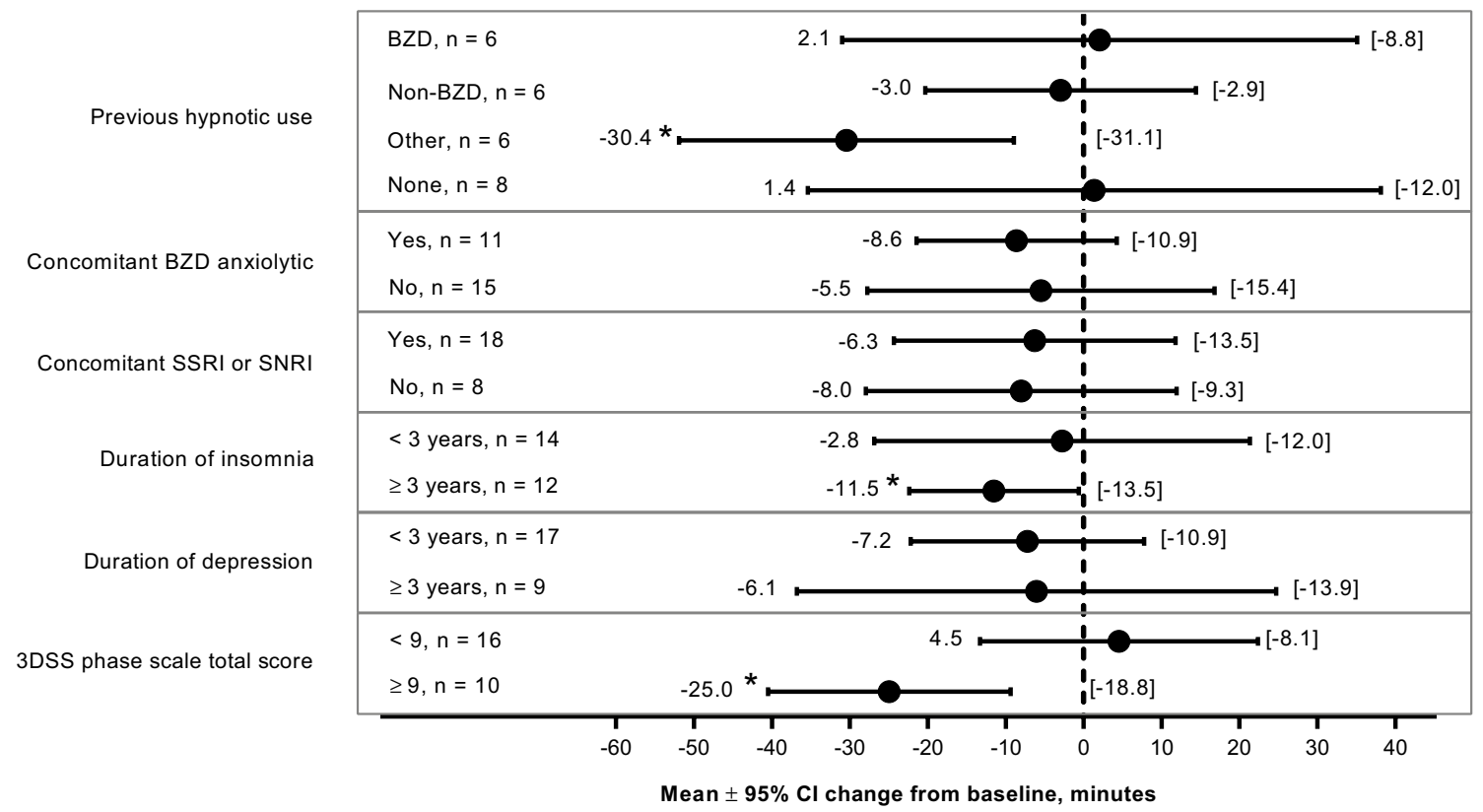

Fig. 2 Change in actigraphy-measured sleep latency by subgroup. Mean changes from baseline and $95 \%$ confidence intervals are plotted [median changes from baseline are shown in square brackets]. *Onesample $t$ test for mean change from baseline; $p<.05$. 3DSS 3-Dimen-

actigraphy-measured sleep latency was largest in the subgroup of patients with regular sleeping habits at baseline, indicated by a 3DSS phase scale total score of $\geq 9$ at baseline (mean \pm SD decrease $-25.0 \pm 21.78 \mathrm{~min} ; p=.0055)$, and in patients with previous use of hypnotics other than benzodiazepines or nonbenzodiazepines (suvorexant, $n=5$; etizolam, $n=1)(-30.4 \pm 20.49 \mathrm{~min} ; p=.0149)$. sional Sleep Scale, BZD benzodiazepine, $C I$ confidence interval, SNRI serotonin and norepinephrine reuptake inhibitor, SSRI selective serotonin reuptake inhibitor

\section{Other sleep assessments}

Based on 3DSS scores, patients reported statistically significant mean improvements from baseline to end of treatment in sleep quality and sleep quantity, but no change in sleep phase (i.e., regularity) (Table 3). The majority of patients (23 patients; $88.5 \%$ ) reported an improvement in their condition at end of treatment based on the PGI (1 patient [3.8\%] very much improved; 16 patients [61.5\%] much improved; 
Table 3 3DSS total score

\begin{tabular}{llllr}
\hline 3DSS category & \multicolumn{4}{l}{ Ramelteon $8 \mathrm{mg}(N=26)$} \\
\cline { 2 - 5 } & Baseline & End of treatment & Change from baseline & $p$ value $^{\mathrm{a}}$ \\
\hline Phase scale total score & $7.2 \pm 3.12$ & $7.2 \pm 2.80$ & $.0 \pm 2.09$ & .9259 \\
Quality scale total score & $5.4 \pm 2.43$ & $8.5 \pm 3.40$ & $3.1 \pm 3.25$ & $<.0001$ \\
Quantity scale total score & $4.8 \pm 2.69$ & $7.0 \pm 3.61$ & $2.3 \pm 3.21$ & .0013 \\
\hline
\end{tabular}

Variables are presented as mean \pm standard deviation

3DSS 3-Dimensional Sleep Scale

${ }^{a}$ One-sample $t$ test for change from baseline to end of treatment

Table 4 HAM-D17 total score

\begin{tabular}{llllr}
\hline HAM-D17 category & \multicolumn{2}{l}{ Ramelteon $8 \mathrm{mg}(N=26)$} & \\
\cline { 2 - 5 } & Baseline & End of treatment & Change from baseline & $p$ value $^{\mathrm{a}}$ \\
\hline Total score & $9.2 \pm 2.12$ & $5.2 \pm 3.20$ & $-4.0 \pm 2.71$ & $<.0001$ \\
Total score, not sleep-related & $4.8 \pm 1.98$ & $3.5 \pm 2.44$ & $-1.3 \pm 2.11$ & .0051 \\
Total score, sleep-related & $4.4 \pm .90$ & $1.7 \pm 1.52$ & $-2.7 \pm 1.82$ & $<.0001$ \\
\hline
\end{tabular}

Variables are presented as mean \pm standard deviation

HAM-D17 17-item Hamilton Rating Scale for Depression

${ }^{\mathrm{a}}$ One-sample $t$ test for change from baseline to end of treatment

6 patients [23.1\%] a little improved); 2 patients $(7.7 \%)$ reported no change and 1 patient (3.8\%), who discontinued ramelteon due to an adverse event of somnolence, considered their condition to be a little worse.

\section{Depression}

Although only patients with mild depression (HAMD17 total score of $\leq 16$ ) were enrolled, mean HAM-D17 total scores improved from baseline to end of treatment (mean \pm SD decrease $-4.0 \pm 2.71 ; p<.0001$ ) (Table 4). Statistically significant improvements were seen whether considering only sleep-related HAM-D17 scores (mean \pm SD decrease $-2.7 \pm 1.82 ; p<.0001)$ or non-sleep-related HAMD17 scores $(-1.3 \pm 2.11 ; p=.0051)$.

\section{Safety}

Nine patients (34.6\%) experienced an adverse event (Table 5). The most common events were viral upper respiratory tract infections (19.2\%) and somnolence (15.4\%). One patient experienced moderate somnolence which led to discontinuation of ramelteon; the event was considered study drug-related and was resolving by the end of the study. All other adverse events were of mild intensity and resolved without intervention. There were no deaths or serious adverse events.
Table 5 Adverse events

\begin{tabular}{ll}
\hline MedDRA preferred term & $\begin{array}{l}\text { Ramelteon } \\
8 \mathrm{mg}(N=26) \\
n(\%)\end{array}$ \\
\hline Any adverse events & $9(34.6)$ \\
Viral upper respiratory tract infection & $5(19.2)$ \\
Somnolence & $4(15.4)$ \\
Dizziness & $1(3.8)$ \\
Pyrexia & $1(3.8)$ \\
\hline
\end{tabular}

MedDRA Medical Dictionary for Regulatory Activities

There were no clinically significant changes in weight or body mass index during the study. No pregnancies were reported.

\section{Discussion}

This is the first study to objectively and subjectively measure the efficacy of ramelteon in the treatment of insomnia in patients with concurrent depression. Patients experienced significant improvements in TST and number of nocturnal awakenings when assessed subjectively using the sleep diary, as well as significant improvements in PGI and 3DSS, suggesting patient benefit. Improvements in sleep latency, whether measured subjectively by sleep diary or objectively by actigraphy, did not achieve statistical significance; however, this exploratory study was not powered to detect statistically significant improvements in sleep parameters. 
Both actigraphy and sleep diary showed mean decreases in sleep latency after 8 weeks of ramelteon treatment (6.8 $\mathrm{min}$ and $11.5 \mathrm{~min}$, respectively), but neither change reached statistical significance. Sleep latency showed high day-to-day variability for most patients, suggesting inconsistent sleep-wake cycles in these non-hospitalized patients which may have impacted the efficacy endpoints. In a phase $2 / 3$ study in Japanese adults with chronic insomnia, ramelteon $8 \mathrm{mg}$ demonstrated statistically significant efficacy in a subgroup analysis of patients whose fluctuation in subjective sleep latency at baseline was within $\pm 30 \mathrm{~min}$ [16]. This may indicate that the assessment of sleep parameters in patients with significant fluctuation in sleep latency at baseline might be unreliable, and therefore, excluding these patients could improve detection of treatment effects. The population in the current study also exhibited relatively mild difficulty in falling asleep, with a mean sleep latency at baseline that was shorter (44.8 min by actigraphy; $51.2 \mathrm{~min}$ by sleep diary) compared with Japanese patients with chronic insomnia in a phase 2 ramelteon study using objective and subjective measurements (61.8 $\mathrm{min}$ by polysomnography; $74.1 \mathrm{~min}$ by sleep diary) [14]. Nevertheless, a significant improvement in actigraphy-measured sleep latency (mean decrease $25.0 \mathrm{~min} ; p=.0055$ ) was seen in the subgroup of patients with a 3DSS total phase score of $\geq 9$, a score indicating a regular sleep pattern. This improvement in sleep latency in patients without a disturbed sleep rhythm emphasizes the role of ramelteon $(8 \mathrm{mg} /$ day $)$ in sleep induction rather than sleep rhythm re-entrainment.

Other subjective assessments used in this study indicated statistically significant mean improvements in sleep parameters at the end of ramelteon treatment, including diarymeasured TST and number of nocturnal awakenings, and 3DSS total scores for sleep quality and quantity. In addition, $88.5 \%$ of patients reported improvement in the PGI. Only the 3DSS total phase score showed no change following ramelteon treatment, which may be because a high proportion of patients ( 10 of 26 patients) already had "good" phase scores of $\geq 9$ at baseline.

Objective actigraphy assessments of change in sleep latency and TST produced similar results to those obtained using the subjective sleep diary method, as supported by the positive Spearman correlation coefficients. However, in contrast to the sleep diary method, the actigraphymeasured mean increase in TST did not reach statistical significance. Moreover, changes in the mean number of nocturnal awakenings measured by actigraphy were not consistent with the results obtained using the sleep diary. Actigraphy-derived sleep data are highly correlated with polysomnography data [21]; however, actigraphy has been shown to provide more consistent sleep measurements in a sleep laboratory setting than at home [24]. We consider the outpatient setting of this study as a strength in that it was designed to reflect real-world experience. However, the outpatient setting may have influenced the precision of the actigraphy assessments. In addition, despite being worn at the waist to reduce artifacts from movements in the extremities, the actigraph may have been influenced by exacerbations of restless legs syndrome and periodic limb movement disorder, which have been associated with some antidepressants [25]. The study is also limited by the small number of enrolled patients, and the lack of a placebo or active comparator group means that changes in symptoms due to expectancy, regression to the mean, or the Hawthorne effect cannot be excluded.

The statistically significant improvement in HAM-D17 total score is meaningful for this population of patients with insomnia and concurrent depression, particularly as the improvement remained significant when excluding sleep-related scores, suggesting that improved sleep may have beneficial effects for depression. The addition of the nonbenzodiazepine eszopiclone in patients receiving fluoxetine for major depressive disorder has also been shown to reduce HAM-D17 scores, as well as improve sleep [6].

Owing to the association between depression and insomnia, any residual sleep disruption after the treatment of depression may increase the likelihood of depressive symptom recurrence. Both shortened and extended sleep duration have been associated with an increased risk of depression, although no causal relationship has been established [26, 27]. In elderly subjects in Japan, those with sleep-onset insomnia were more likely to experience depression 3 years later [28]. Benzodiazepines are often used concomitantly with antidepressants to treat anxiety, and have been reported to be as effective as monotherapy in the treatment of anxious depression [29]. As sleep-onset insomnia often correlates with anxiety, anxiolytic benzodiazepines may potentially shorten sleep latency. However, the sedative and anxiolytic effects of benzodiazepine and nonbenzodiazepine hypnotics limit their use in some patients with depression. Insomnia in depression requires long-term treatment, but benzodiazepine dependency can develop quickly and so should be used cautiously as a long-term option [30]. No dependency was observed with ramelteon after 24 weeks of use in a longterm clinical trial in Japan. Hypnotics are also associated with rebound worsening of anxiety and insomnia following their discontinuation, as well as a withdrawal syndrome characterized by feelings of malaise [11]. An alternative option, the orexin receptor antagonist suvorexant, has a long half-life and is associated with challenging residual next-day somnolence in some patients [31]. Our study did not reveal any major concerns relating to residual effects with ramelteon. One patient discontinued ramelteon use due to moderate somnolence; all other adverse events were mild and resolved without intervention. Ramelteon $8 \mathrm{mg}$ once daily was well tolerated when 
coadministered with antidepressants for an 8-week period and no new safety signals were detected.

The use of benzodiazepine and nonbenzodiazepine hypnotics might be inappropriate in those depressive patients who are vulnerable to their muscle relaxant or amnesic side effects. There is limited evidence for the use of ramelteon to treat insomnia in patients with mental disorders. Although this was an exploratory study, the results suggest that ramelteon may be efficacious for sleep-onset insomnia in this patient population.

\section{Acknowledgements Role of the sponsor}

Takeda Pharmaceutical Company Limited was involved in the study design, data collection, data analysis, and preparation of the manuscript.

\section{Role of contributors}

All authors have seen and approved the manuscript. All authors participated in data collection, the interpretation of study results, and in the drafting and critical revision of the manuscript. NU and MU were involved in the study design. $\mathrm{KN}$ and $\mathrm{KM}$ conducted the statistical analysis.

\section{Other contributors/acknowledgements}

The authors would like to thank the patients who participated in this study, as well as Takuma Endo (former employee of Takeda Pharmaceutical Company Limited) for contributing to study design and study start-up, and Junko Kunisa (A2 Healthcare Corporation) for data analysis.

Funding This study was sponsored by Takeda Pharmaceutical Company Limited, manufacturer and licensee of ramelteon. Medical writing assistance was provided by Linda Donnini, Ph.D. and Serina Stretton, Ph.D., CMPP of ProScribe-Envision Pharma Group, and was funded by Takeda Pharmaceutical Company Limited. ProScribe's services complied with international guidelines for Good Publication Practice (GPP3).

\section{Compliance with ethical standards}

Conflicts of interest KN and KM are employees of Takeda Pharmaceutical Company Limited. NU and MU have participated in Advisory Panels and Speaker's Bureaus for Takeda Pharmaceutical Company Limited.

Ethical committee permission The study was approved by the ethics committees of each of the 7 study sites.

Informed consent Informed consent was obtained from all individual participants included in the study.

Research involving human participants and/or animals All procedures performed in studies involving human participants were in accordance with the ethical standards of the institutional and/or national research committee and with the 1964 Helsinki declaration and its later amendments or comparable ethical standards.

Open Access This article is distributed under the terms of the Creative Commons Attribution 4.0 International License (http://creativeco mmons.org/licenses/by/4.0/), which permits unrestricted use, distribution, and reproduction in any medium, provided you give appropriate credit to the original author(s) and the source, provide a link to the Creative Commons license, and indicate if changes were made.

\section{References}

1. Furihata R, Uchiyama M, Takahashi S, Suzuki M, Konno C, Osaki K, Konno M, Kaneita Y, Ohida T, Akahoshi T, Hashimoto $\mathrm{S}$, Akashiba T. The association between sleep problems and perceived health status: a Japanese nationwide general population survey. Sleep Med. 2012;13:831-7.

2. Fushimi M. Prevalence of depressive symptoms and related factors in Japanese employees: a comparative study between surveys from 2007 and 2010. Psychiatry J. 2015;2015:537073.

3. Mahlich J, Tsukazawa S, Wiegand F. Estimating prevalence and healthcare utilization for treatment-resistant depression in Japan: a retrospective claims database study. Drugs Real World Outcomes. 2018;5:35-43.

4. Doi Y, Minowa M, Okawa M, Uchiyama M. Prevalence of sleep disturbance and hypnotic medication use in relation to sociodemographic factors in the general Japanese adult population. J Epidemiol. 2000;10:79-86.

5. Sunderajan P, Gaynes BN, Wisniewski SR, Miyahara S, Fava M, Akingbala F, DeVeaugh-Geiss J, Rush AJ, Trivedi MH. Insomnia in patients with depression: a STAR*D report. CNS Spectr. 2010;15:394-404.

6. Fava M, McCall WV, Krystal A, Wessel T, Rubens R, Caron J, Amato D, Roth T. Eszopiclone co-administered with fluoxetine in patients with insomnia coexisting with major depressive disorder. Biol Psychiatry. 2006;59:1052-60.

7. Driesen K, Jansen NW, van Amelsvoort LG, Kant I. The mutual relationship between shift work and depressive complaintsa prospective cohort study. Scand J Work Environ Health. 2011;37:402-10.

8. Emens J, Lewy A, Kinzie JM, Arntz D, Rough J. Circadian misalignment in major depressive disorder. Psychiatry Res. 2009;168:259-61.

9. Roehrs T, Roth T. Insomnia pharmacotherapy. Neurotherapeutics. 2012;9:728-38.

10. Nakamura M, Inoue Y. Benzodiazepine and nonbenzodiazepine hypnotics. Nihon Rinsho. 2015;73:1010-5 (in Japanese).

11. Lader M. Anxiety or depression during withdrawal of hypnotic treatments. J Psychosom Res. 1994;38(Suppl 1):113-23 (discussion 8-23).

12. Miyamoto M. Pharmacology of ramelteon, a selective MT1/ MT2 receptor agonist: a novel therapeutic drug for sleep disorders. CNS Neurosci Ther. 2009; 15:32-51.

13. Kato K, Hirai K, Nishiyama K, Uchikawa O, Fukatsu K, Ohkawa S, Kawamata Y, Hinuma S, Miyamoto M. Neurochemical properties of ramelteon (TAK-375), a selective MT1/MT2 receptor agonist. Neuropharmacology. 2005;48:301-10.

14. Kohsaka M, Kanemura T, Taniguchi M, Kuwahara H, Mikami A, Kamikawa K, Uno H, Ogawa A, Murasaki M, Sugita Y. Efficacy and tolerability of ramelteon in a double-blind, placebocontrolled, crossover study in Japanese patients with chronic primary insomnia. Expert Rev Neurother. 2011;11:1389-97.

15. Liu J, Clough SJ, Hutchinson AJ, Adamah-Biassi EB, PopovskaGorevski M, Dubocovich ML. MT1 and MT2 melatonin receptors: a therapeutic perspective. Annu Rev Pharmacol Toxicol. 2016;56:361-83.

16. Uchimura N, Ogawa A, Hamamura M, Hashimoto T, Nagata H, Uchiyama M. Efficacy and safety of ramelteon in Japanese adults with chronic insomnia: a randomized, double-blind, placebo-controlled study. Expert Rev Neurother. 2011;11:215-24. 
17. Uchiyama M, Hamamura M, Kuwano T, Nishiyama H, Nagata $\mathrm{H}$, Uchimura N. Evaluation of subjective efficacy and safety of ramelteon in Japanese subjects with chronic insomnia. Sleep Med. 2011;12:119-26.

18. Neubauer DN. A review of ramelteon in the treatment of sleep disorders. Neuropsychiatr Dis Treat. 2008;4:69-79.

19. Gross PK, Nourse R, Wasser TE. Ramelteon for insomnia symptoms in a community sample of adults with generalized anxiety disorder: an open label study. J Clin Sleep Med. 2009;5:28-33.

20. Ueda H. Clinical usefulness of ramelteon in the treatment of insomnia with mental disorder. Jpn J Clin Psychopharmacol. 2014;17:65-73.

21. Nakazaki K, Kitamura S, Motomura Y, Hida A, Kamei Y, Miura N, Mishima K. Validity of an algorithm for determining sleep/wake states using a new actigraph. J Physiol Anthropol. 2014;33:31.

22. Matsumoto Y, Uchimura N, Ishida T, Morimatsu Y, Mori M, Inoue M, Kushino N, Hoshiko M, Ishitake T. The relationship of sleep complaints risk factors with sleep phase, quality, and quantity in Japanese workers. Sleep Biol Rhythms. 2017;15:291-7.

23. Zimmerman M, Martinez JH, Young D, Chelminski I, Dalrymple K. Severity classification on the Hamilton Depression Rating Scale. J Affect Disord. 2013;150:384-8.

24. McCall C, McCall WV. Objective vs subjective measurements of sleep in depressed insomniacs: first night effect or reverse first night effect? J Clin Sleep Med. 2012;8:59-65.

25. Kolla BP, Mansukhani MP, Bostwick JM. The influence of antidepressants on restless legs syndrome and periodic limb movements: a systematic review. Sleep Med Rev. 2018;38:131-40.
26. Kaneita Y, Ohida T, Uchiyama M, Takemura S, Kawahara K, Yokoyama E, Miyake T, Harano S, Suzuki K, Fujita T. The relationship between depression and sleep disturbances: a Japanese nationwide general population survey. J Clin Psychiatry. 2006;67:196-203.

27. Zhai L, Zhang H, Zhang D. Sleep duration and depression among adults: a meta-analysis of prospective studies. Depress Anxiety. 2015;32:664-70.

28. Yokoyama E, Kaneita Y, Saito Y, Uchiyama M, Matsuzaki Y, Tamaki T, Munezawa T, Ohida T. Association between depression and insomnia subtypes: a longitudinal study on the elderly in Japan. Sleep. 2010;33:1693-702.

29. Benasi G, Guidi J, Offidani E, Balon R, Rickels K, Fava GA. Benzodiazepines as a monotherapy in depressive disorders: a systematic review. Psychother Psychosom. 2018;87:65-74.

30. Bushnell GA, Stürmer T, Gaynes BN, Pate V, Miller M. Simultaneous antidepressant and benzodiazepine new use and subsequent long-term benzodiazepine use in adults with depression, United States, 2001-2014. JAMA Psychiatry. 2017;74:747-55.

31. Herring WJ, Roth T, Krystal AD, Michelson D. Orexin receptor antagonists for the treatment of insomnia and potential treatment of other neuropsychiatric indications. J Sleep Res. 2018;28:e12782.

Publisher's Note Springer Nature remains neutral with regard to jurisdictional claims in published maps and institutional affiliations. 